\title{
Treatment of Influenza-Induced Acute Lung Injury with Iron Oxide Nanoparticles using an Ischemic-Reperfusion Model
}

\author{
K. Chew ${ }^{1}$, A. Waldron ${ }^{2}$, C. Queenan ${ }^{2}$, R. Pergolizzi ${ }^{1}$, and T. Hoffmann ${ }^{3}$ \\ 1. Bergen County Academies, Stem Cell Research Lab, 200 Hackensack Avenue, Hackensack, NJ 07601 \\ 2. Bergen County Academies, Nano-Structural Imaging Lab, 200 Hackensack Avenue, Hackensack NJ \\ 07601 \\ 3. Englewood Hospital and Medical Center, Surgical Research Laboratory, 350 Engle Street, Englewood \\ NJ, 07631
}

Influenza is a highly contagious viral infection. To prevent complications such as pneumonia, a better treatment for lung inflammation must be found. One potentially effective solution may be to utilize iron oxide nanoparticles (ION). Nanoparticles have become increasingly used in medicine. Specifically, ION have been studied for their potential in influenza diagnosis [1]. Some studies have shown that ION assist in stabilizing inflammation in certain cell lines [2], while other studies have used ION to induce inflammation [3]. The interaction between ION and human monocyte-macrophages (HMMs) has also been studied [4]. HMMs are involved inflammatory signaling within a cell. It was found that ION were retained within HMM lysosomes, which prevented them from inducing inflammatory cytokines.

In this study, it was hypothesized that ION stabilize inflammation. When the influenza virus enters a lung cell, the inflammatory process is initiated. As a result, the protein tumor necrosis factor alpha (TNF- $\alpha$ ) is secreted [5]. Additionally, nitric oxide acts as a mediator in the inflammatory process; it is consumed as inflammation increases. Therefore, cells studied in vitro that are stimulated with TNF- $\alpha$ would produce lower levels of nitric oxide as compared to cells stimulated with ION [6]. It was also hypothesized that ION would be non-toxic to the proliferation of cells; cells treated with higher concentrations of ION would have increased cell viability than those treated with lower concentrations of ION.

This hypothesis can also be studied in vivo. A super mesenteric artery (SMA) ischemia reperfusion procedure mimics the mechanism that causes TNF- $\alpha$ secretion after interaction with influenza virus. Lipid peroxidation, overproduction of nitric oxide, and other complex mechanisms caused by SMA ischemia reperfusion results in lung injury [7]. In lung samples, the anatomical distance between each alveoli sac can be measured [8]; a large distance between each alveoli sac indicates the collapse of the sacs, leading to capillary obstruction, which is a mark of inflammation [9]. Additionally, the number of edema-filled sacs within a lung is a further indicator of inflammation [10]. Therefore, in vivo samples obtained after ION inhalation should indicate a reduced amount of lung injury, which can be determined by measuring the distance between alveoli sacs and calculating the number of edema filled sacs.

For in vitro studies, application of ION to lung cells stimulated with TNF- $\alpha$ showed increased levels of nitric oxide, indicating a decrease in inflammation. At high concentrations of ION, nitric oxide secretion nearly matched that present in cells without TNF- $\alpha$ treatment. Additionally, cell viability in a 48-hour period was consistent for all concentrations of ION, suggesting that the nanoparticles were not cytotoxic. For in vivo studies, there was a statistically significant difference in the level of lung injury between samples that inhaled saline and samples that inhaled ION. Overall, ION show promise as an effective treatment for influenza-induced lung injury [11]. 


\section{References}

[1] T. Chou, et al., Journal of Nanobiotechnology 9 (2011) 1-13.

[2] A. Jefferson, et al., Theranostics 3 (2013) 428.

[3] A. Elder, et al., In Nanotechnology and the Environment: Applications and Implications Progress Review Workshop III 10 (2005) 105.

[4] K. Müller, et al., Biomaterials 28 (2007) 1629-1642.

[5] I. Julkunen et al., Cytokine \& growth factor reviews 12 (2001) 171-180.

[6] J. Jiang, et al., Nitric Oxide 25 (2011) 275-281.

[7] K. Liu, et al., World Journal of Gastroenterology 13 (2007) 299.

[8] J. Warren, et al., Journal of Clinical Investigation 84 (1989) 1873.

[9] M. McElroy, et al., European Respiratory Journal 24 (2004) 664-673.

[10] L. Colletti, et al., Journal of Clinical Investigation 85 (1990) 1936.

[11] The authors would like to acknowledge the administration of the Bergen County Technical

Schools for their continued support of the research program.

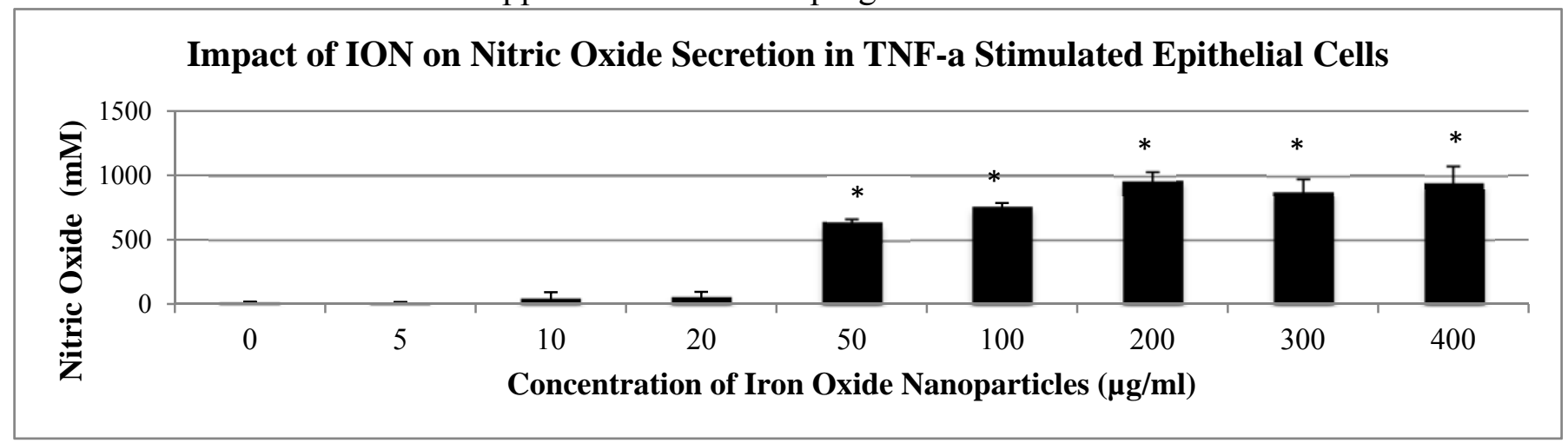

Figure 1: Nitric oxide levels in epithelial cell cultures treated with TNF- $\alpha$ for 24 hours, followed by incubation with nanoparticles for 2 hours. Data is represented by mean $\pm \operatorname{SD}(n=3)$. Asterisk indicates statistical significance as compared to control $(\mathrm{p}<0.005)$.
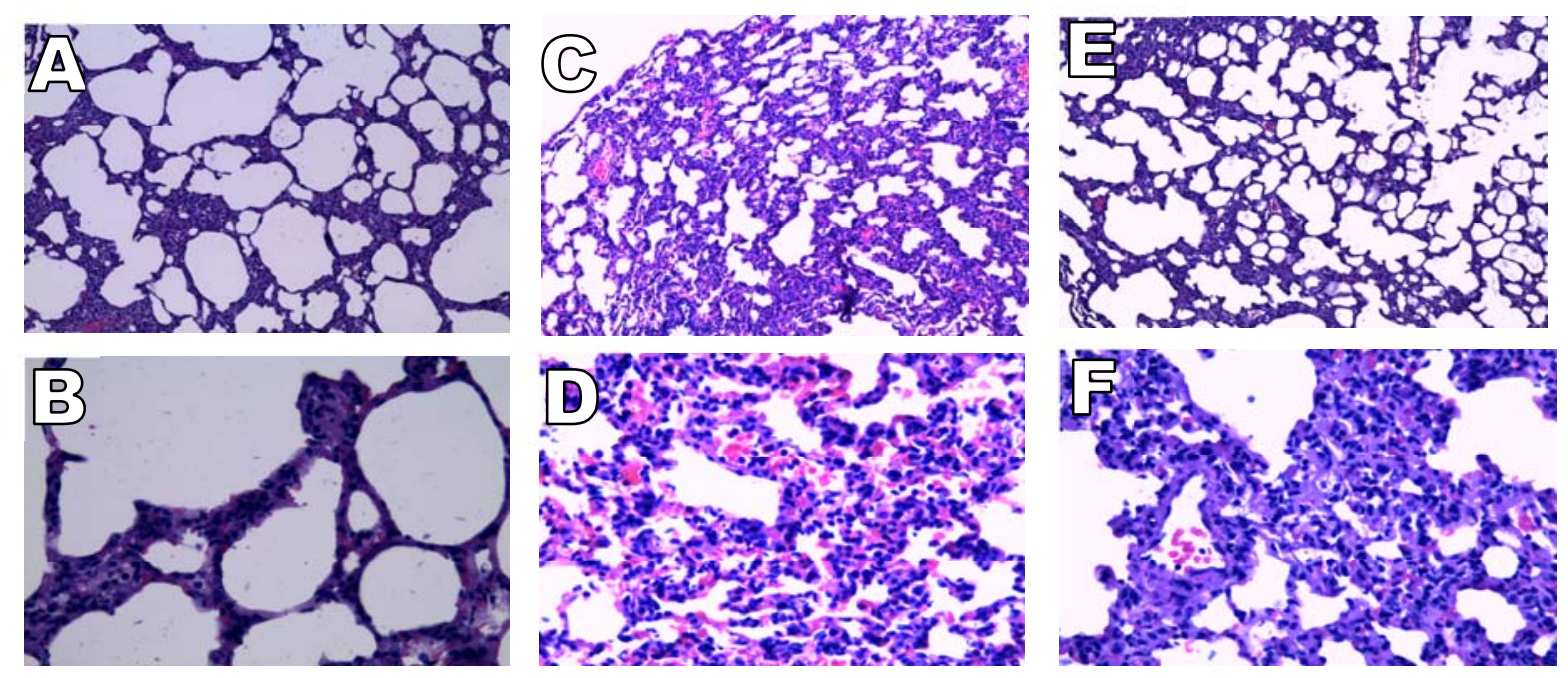

Figure 2: Light micrographs of microthin sections of lung tissue stained with hematoxylin and eosin. Images A, C, and E acquired at 100X magnification. Images B, D, and F acquired at $400 \mathrm{X}$ magnification. Images $A$ and $B$ : normal lung tissue. Images $C$ and D: lung tissue after ischemia reperfusion (IR) and inhaling saline. Images $\mathrm{E}$ and $\mathrm{F}$ : lung tissue after the IR and inhaling ION. 